\title{
ANÁLISIS DEL CRECIMIENTO DE CINCO HÍBRIDOS DE ZANAHORIA (Daucus carota L.) MEDIANTE LA METODOLOGÍA DEL ANÁLISIS FUNCIONAL ${ }^{1}$
}

\author{
Tatiana Vega Rojas ${ }^{* *}$, Carlos Méndez Soto ${ }^{2 / *}$, Werner Rodríguez Montero* \\ Palabras clave: Zanahoria, híbridos, análisis de crecimiento, fenología. \\ Keywords: Carrot, hybrids, plant growth analysis, phenology. \\ Recibido: 11/07/11 \\ Aceptado: 03/12/11
}

\begin{abstract}
RESUMEN
El análisis de crecimiento de 5 híbridos de zanahoria (Daucus carota L.) se efectuó en Cipreses de Oreamuno, Cartago, con el objetivo de describir el crecimiento y la fenología del cultivo de zanahoria. El análisis de crecimiento se realizó mediante la metodología de análisis funcional. Se evaluó el área foliar y peso seco total de cada órgano de la planta, se ajustaron modelos de regresión no lineal para dichas variables, se obtuvieron los parámetros que definen las funciones matemáticas que describen el crecimiento y se representó gráficamente cada modelo para cada variable evaluada. Las variables de peso seco total y de raíz se ajustaron en la función logística no-lineal asintótica: PS=alfa/(1+exp(beta(x-gamma))); el peso seco de hojas y el área foliar se ajustaron en la función tipo "campana": $\mathrm{PS}=$ alfa*exp $\left(-\right.$ beta $\left.(\mathrm{x}-\mathrm{gamma})^{2}\right)$. Las plantas presentaron una curva de crecimiento sigmoidal con fases bien definidas: una fase de crecimiento lento, luego una etapa de crecimiento exponencial, seguida de una etapa de disminución del crecimiento y por último una etapa de estabilidad. La planta dedica sus primeros estados de
\end{abstract}

\footnotetext{
1 Este trabajo forma parte de la tesis de grado del primer autor.

2 Autor para correspondencia. Correo electrónico: carlos.mendez@ucr.ac.cr
}

\begin{abstract}
Plant growth analysis of five hybrids of carrot (Daucus carota L.) by functional analysis methodology. Plant growth analysis of 5 hybrids of carrot (Daucus carota L.) was conducted in Cipreses, Oreamuno, Cartago, with the aim to describe the phenology and growth of carrot. Growth analysis was performed using the methodology of functional analysis. The leaf area and the dry weight of each organ and total plant were evaluated, non-linear regression models were adjusted for these variables, the parameters that define the mathematical functions that best describe the growth were obtained and each model was plotted for each variable evaluated. The roots and total dry weight variables were adjusted to an asymptotic nonlinear logistic function: $\mathrm{PS}=$ alpha/ (1+exp (-beta (x-gamma))); the leaves dry weight and the leaf area were adjusted into the belltype function: PS=alpha*exp (-beta $\left.(\mathrm{x} \text {-gamma })^{2}\right)$. Plants showed a sigmoidal growth curve with well-defined stages: a slow growth phase, then an exponential growth segment, followed by a period of reduced growth and finally a stage of stability. The plant spends its early stages
\end{abstract}


desarrollo a establecer su maquinaria fotosintética, en competencia directa con el desarrollo radical. La raíz comienza a engrosar entre los 63 dds y los 77 dds según el híbrido, durante la etapa II de crecimiento, con una mayor proporción de asimilados destinados a la raíz, lo que conduce a un aumento en su diámetro. Finalmente, del análisis de las variables de peso seco total y área foliar, se puede establecer 2 grupos: por un lado Suprema, Esperanza y Bangor, y por otro CLX3193 y Sirkana. El primer grupo presentó los valores más altos para dichas variables.

\section{INTRODUCCIÓN}

El cultivo de la zanahoria (Daucus carota L., Umbelliferae) en Costa Rica, ha sido considerado por mucho tiempo como un producto menor en cuanto a su producción y consumo. Sin embargo, el interés por este cultivo se ha incrementado dadas las expectativas de exportación, donde hay una ventana para nuevos mercados, más fuentes de trabajo y hasta la posibilidad de constituirse en una opción que contribuya a mejorar la balanza comercial del país.

El crecimiento es un fenómeno cuantitativo que se mide en gramos de materia seca acumulados por unidad de tiempo (Rodríguez y Leihner 2006, Rojas y Rovalo 1985). El término "análisis de crecimiento" se refiere al uso de métodos cuantitativos que describen todo el sistema de la planta con crecimiento bajo condiciones naturales, semi naturales o controladas. El análisis de crecimiento provee la capacidad para interpretar la forma y función de la planta (Hunt 2003).

Rodríguez y Leihner (2006) indican que hay 2 enfoques metodológicos básicos para realizar el análisis de crecimiento: el clásico y el funcional. El primero consiste en practicar pocos muestreos a lo largo del ciclo de cultivo, pero of development establishing the photosynthetic machinery, indirect competition with radical development. The root begins to thicken between 63-77 dap, during the second phase of growth, with a greater proportion of assimilates allocated to the root, leading to an increase in its diameter. Finally, from the analysis of the variables of total dry weight and leaf area, 2 groups can be established: on the one side Supreme, Esperanza and Bangor, and on the other CLX-3193 and Sirkana. The first group showed the highest values for such variables.

cada uno de ellos con muchas repeticiones. Éste es adecuado cuando la meta es la comparación del efecto de tratamientos de interés sobre el crecimiento. El segundo, por el contrario, consta de pocas repeticiones pero muchos momentos de muestreo. Se utiliza para simular el crecimiento mediante el uso de funciones matemáticas que describan adecuadamente el desarrollo de las plantas.

A diferencia del análisis de crecimiento clásico, el análisis funcional utiliza la totalidad de la información recabada para definir cada punto de la curva de crecimiento. En el análisis funcional es necesario tener un gran número de muestreos; si las circunstancias no permiten esto, el análisis funcional no se puede aplicar (Venus y Causton 1979). Los parámetros que definen las funciones matemáticas aludidas por el análisis funcional son determinados mediante el procedimiento estadístico llamado análisis de regresión no-lineal (Rodríguez y Leihner 2006).

Antes de que aparezcan las primeras hojas verdaderas, las plántulas de zanahoria presentan una clara delimitación entre la raíz y el hipocotilo. Con la aparición de las primeras hojas, esta distinción tiende a desdibujarse y las raíces laterales comienzan a desarrollarse alineadas en 4 líneas longitudinales (Tirilly y Bourgeuis 2002). 
A partir de los 18 días después de la siembra (dds) se puede observar una separación entre la raíz de almacenamiento y las raíces fibrosas (Hole y Dearman 1991).

La zanahoria presenta un crecimiento lento en sus primeros estados de desarrollo vegetativo (Richmond 2009 y Suojala 2000). No obstante, Hole et al. (1983), al estudiar el crecimiento de 4 cultivares de zanahoria en invernadero, encontraron que el crecimiento tanto de la parte aérea como la raíz fue exponencial hasta los 60 dds, para decrecer posteriormente. Westerveld et al. (2006), observaron que la acumulación de materia seca en el follaje se incrementa gradualmente hasta un punto máximo o en ocasiones disminuye al final del ciclo. En los casos en que la materia seca del follaje decrece, esta alcanza un máximo entre los 115 y 135 dds, lo que también concuerda con lo reportado por Stanhill (1977) y Suojala (2000).

La radícula origina una raíz principal típica y pivotante, de la cual surgen raíces secundarias para la absorción de nutrimentos y agua (Valadez 1998 y Tirilly y Bourgeuis 2002). Durante los primeros 50 días la raíz de zanahoria crece rápidamente en longitud. Ese crecimiento es considerablemente más rápido que el aumento en el peso de la raíz. La acumulación de materia seca en la raíz es un proceso lento al principio, pero después del primer tercio del ciclo la raíz comienza a aumentar de forma constante hasta la cosecha. Cerca de la cosecha la tasa de ganancia de peso de la raíz disminuye (Plant Protection 2005).

El engrosamiento inicia en la parte superior de la raíz a la punta y continua mientras las hojas provean fotoasimilados (Tirilly y Bourgeuis 2002). El engrosamiento de la raíz es resultado de la actividad del cambium secundario, cuya iniciación depende del aporte de asimilados y reguladores de crecimiento proveniente de las hojas (Benjamin et al. 1997). Las raíces alcanzan su peso máximo a los 120 días después de la emergencia (dde), los siguientes 30 días muestra un aumento pequeño e irregular, lo cual sugiere que el patrón del crecimiento relativo empieza a cambiar entre los 105 y 120 dde (Stanhill 1977 y Suojala 2000).
El crecimiento de la raíz depende de los asimilados provenientes del follaje (Suojala 2000). La raíz es un importante sumidero de asimilados, pues acumula el $40 \%$ de la materia seca producida por la planta a las 9 semanas después de la siembra (Benjamin y Wren 1978).

Desde las primeras etapas de crecimiento, tanto la parte aérea como la raíz de la zanahoria se desarrollan simultáneamente y en sincronía. Inicialmente domina el crecimiento de la parte aérea, cuando el sistema foliar alcanza su pleno desarrollo se acelera el crecimiento de la raíz principal en detrimento del follaje, lo que sugiere un aumento en la movilización de asimilados hacia la raíz (Hole et al. 1983, Tirilly y Bourgeuis 2002 y Krzesiński y Knaflewski 2004).

La partición de asimilados entre la parte aérea y la raíz, durante el ciclo de cultivo, es una expresión de la interacción entre los factores genéticos, los ambientales y los culturales (Hole et al. 1983, Bertsch 1998 y Suojala 2000).

Al haberse considerado durante largo tiempo a la zanahoria como un producto de poca importancia, no se cuenta con información actualizada sobre su manejo agronómico; por lo que debe desarrollarse las bases científicas que permitan generar la tecnología para mejorar y optimizar la producción de zanahoria. El objetivo del presente trabajo es describir la fenología y el crecimiento de 5 híbridos de zanahoria en la zona alta de Cartago.

\section{MATERIALES Y MÉTODOS}

El análisis de crecimiento se realizó de marzo a julio del 2009, en Cipreses de Oreamuno, Cartago, coordenadas geográficas $9^{\circ} 533^{\prime} 5,6^{\prime \prime} \mathrm{N}$, $83^{\circ} 50^{\prime} 39,0^{\prime \prime}$ O, a una altitud de $1721 \mathrm{msnm}$, en la empresa agrícola del Ing. Agr. Antonio Castro, con seguimiento de sus prácticas agrícolas.

El suelo es de origen volcánico, con características de Andisol, por lo que sus propiedades físicas son adecuadas para el cultivo de zanahoria. El suelo no presentaba problemas de acidez, sin embargo mostró niveles bajos de $\mathrm{Mg}, \mathrm{K}, \mathrm{P}$, Zn y Mn. 
Los híbridos utilizados fueron Bangor (Bejo Zaden B.V) por ser el híbrido con mayor área de siembra en el país, Esperanza (Seminis Vegetable Seeds, Inc.) por resultar un material promisorio del trabajo realizado por Richmond 2009 y Suprema (Isla Sementes), CLX-3193 F1 (Clause) y Sirkana (Nunhems) como nuevos materiales comerciales promisorios.

La preparación del terreno se realizó 15 días antes de la siembra. El terreno se encaló con hidróxido de calcio a una dosis de $2 \mathrm{Tm}_{\mathrm{T}} \mathrm{ha}^{-1}$. La semilla fue colocada a mano, debido a las diferencias en el calibre de la semilla en cada híbrido. Dado que la zanahoria presenta una germinación muy variable, se procedió a colocar aproximadamente 55 semillas en cada hilera de siembra de un metro de longitud, con el objetivo de obtener al final 30 plantas por surco de siembra.

A la siembra se aplicó el nematicida Nemacur 5 GR (Fenamifos) a una dosis de 43 kg.ha ${ }^{-1}$ para el control de nematodos como Meloidogyne. También se realizó una aplicación en drench de los siguientes fungicidas: Proplant 72,2 SL (propamocarb) a $2,5 \mathrm{ml}^{-\mathrm{l}^{-1}}$ + Carbendazina $50 \mathrm{SC}$ (Carbendazina) a 2,5 ml. $\mathrm{l}^{-1}+$ Banrot (Etridiazole + Metilthiophanato) a 1 gr. l $^{-1}$, para el control de hongos de suelo como Rizoctonia sp., Fusarium sp., Phytophthora sp., y Pythium sp.

Ocho días después de la siembra (dds) se realizó una aplicación de insecticida Cipermetrina 25 EC (Cipermetrina) a $0,625 \mathrm{ml}^{-\mathrm{l}^{-1}}+$ Tamaron $60 \mathrm{SL}$ (Metamidofos) a 1,25 ml. $\mathrm{l}^{-1}$ para el control de Agrotis spp. A los 15 dds se aplicó Cipermetrina 25 EC (Cipermetrina) a 0,625 ml. $.^{-1}+$ Vydate
$24 \mathrm{SL}$ (Oxamyl) a $5 \mathrm{ml} . \mathrm{l}^{-1}$ para control de gusanos cortadores y nematodos. A los 22 dds se aplicaron el insecticida Cipermetrina 25 EC (Cipermetrina) a $0,625 \mathrm{ml} . \mathrm{l}^{-1}+$ los fungicidas Banrot (Etridiazole + Metilthiophanato) a 1 gr. $.^{-1}+$ Moncut $50 \mathrm{WP}$ (Flutolanil) a 2,5 gr. l $^{-1}$ para el control de gusanos cortadores y hongos de suelo respectivamente, adicionalmente se aplicó Vydate 24 SL (Oxamyl) a $5 \mathrm{ml}^{-\mathrm{l}^{-1}}$ para el control de nematodos. Para el control de Liryomiza se realizaron 2 aplicaciones de Vertimec 1,8 EC (Abamectina) a 0,5 $\mathrm{ml}^{-1}$.

Para el control de los hongos que afectan el follaje, Alternaria dauci y Cercospora carotae se realizaron aplicaciones de fungicidas cada 8 días con las siguientes prácticas:

1. Bellis 38 WG (Boscalid+Pyraclostrobin) a 1 gr. $^{-1}+$ Antracol 70 WP (Propineb) a 3,75 gr. $1^{-1}$.

2. Kal-sil $72 \mathrm{SC}$ (Clorotalonil) a $2,5 \mathrm{ml} . \mathrm{l}^{-1}+$ Carbendazina 50 SC (Carbendazina) a 2,5 ml. $\mathrm{l}^{-1}$.

3. Calidan 27,5 SC (Iprodione + Carbendazina) a 2,5 ml. $1^{-1}$ + Bactericida Agri-Mycin 16,5 WP (Oxitetraciclina + Estreptomicina) a 1 gr. ${ }^{-1}$.

4. Kal-sil $72 \mathrm{SC}$ (Clorotalonil) a $2,5 \mathrm{ml}^{-1^{-1}+}$ Carbendazina 50 SC (Carbendazina) a 2,5 ml. $1^{-1}$.

Se aplicó el herbicida Afalon 45 SC (Linuron) a $1,25 \mathrm{mll}^{-1}$ para el control de malezas, 8 y 15 días después de la primera fertilización.

El plan de fertilización aplicado se presenta en el Cuadro 1.

Cuadro 1. Total de nitrógeno, fosforo y potasio $\left(\mathrm{kg}_{\mathrm{k}} \mathrm{ha}^{-1}\right)$ aplicados al cultivo de zanahoria, Oreamuno, Cartago, 2009.

\begin{tabular}{cccc}
\hline Fertilización & $\begin{array}{c}\text { Nitrógeno } \\
\left(\mathrm{kg}^{-1} \mathrm{ha}^{-1} \mathrm{de} \mathrm{N}\right)\end{array}$ & $\begin{array}{c}\text { Fósforo } \\
\left(\mathrm{kg}^{-1} \mathrm{ha}^{-1} \mathrm{de} \mathrm{P}_{2} \mathrm{O}_{5}\right)\end{array}$ & $\begin{array}{c}\text { Potasio } \\
\left(\mathrm{kg}^{-} \mathrm{ha}^{-1} \mathrm{de} \mathrm{K}_{2} \mathrm{O}\right)\end{array}$ \\
\hline $1^{\circ}: 30 \mathrm{dds}$ & 61 & 123 & 61 \\
$2^{\circ}: 60 \mathrm{dds}$ & 61 & 56 & 92 \\
Total & 122 & 179 & 153 \\
\hline
\end{tabular}


No se observaron deficiencias nutricionales en el cultivo, sin embargo como complemento a la fertilización al suelo se hicieron aplicaciones foliares de N, P, K, Mg, Ca y elementos menores.

El análisis de crecimiento se realizó mediante la metodología de análisis funcional (Rodríguez y Leihner 2006). Se utilizó un diseño experimental de bloques completos al azar con un arreglo de parcelas divididas, donde la parcela grande correspondió a los híbridos y la parcela pequeña a las épocas de muestreo, con 4 repeticiones. La unidad experimental consistió en una parcela de un metro cuadrado (1 m de largo x $1 \mathrm{~m}$ de ancho) con 5 surcos de siembra. Cada surco de siembra con aproximadamente 30 plantas.

Se efectuaron 10 muestreos en total en 2 etapas. En la primera etapa se realizaron 5 muestreos cada 8 días, de 39 a 68 días después de la siembra (dds); cada muestreo incluyó un número de plantas equivalente a un metro cuadrado. En la segunda fase se realizaron 5 muestreos cada 15 días, de 81 a 139 dds; en este caso se muestrearon de 10 a 15 plantas completas de zanahoria.

En cada evaluación se seleccionaron las plantas que representaban el desarrollo fenológico propio de la época de muestreo y se eliminaron las que presentaron algún defecto (raíces bifurcadas, reventadas, hojas con incidencia de Alternaria y Cercospora).

El material cosechado en la parcela fue trasladado a la Estación Experimental Fabio Baudrit Moreno, Barrio San José de Alajuela, para su procesamiento. El material se lavó y se separó en complejo raíz-tallo y follaje. El área foliar se determinó mediante un medidor de área foliar Li-Cor 3100 (Lincoln, Nebraska). Cada órgano fue colocado en bolsas de papel y secado en una estufa a $70^{\circ} \mathrm{C}$ por $48 \mathrm{~h}$ hasta peso constante.

Con el peso seco de las hojas, complejo tallo-raíz y total se determinó las curvas de distribución de materia seca a través del ciclo. Si bien los datos de área foliar y peso seco fueron expresados en $\mathrm{dm}^{2}$. planta $^{-1}$ y g.planta ${ }^{-1}$ respectivamente, estos se transformaron a escala logarítmica para homogenizar su varianza.
Se ajustaron modelos de regresión no lineal para el peso seco de cada órgano, total y del área foliar, para cada híbrido, mediante el procedimiento NLIN de SAS. Se obtuvieron los parámetros que definen las funciones matemáticas de mejor ajuste de los datos para describir el crecimiento y se graficó cada modelo para las variables evaluadas.

Con la primera derivada de cada una de las funciones matemáticas obtenidas, la cual corresponde a la tasa de crecimiento relativo (TCR) del cultivo, se obtuvo la tasa relativa de partición de asimilados.

\section{Ecuación 1}

Partición=TCR (raíz u hojas)/TCR biomasa total

Para evaluar la hipótesis de diferencias en el crecimiento debidas a los híbridos, se estimaron los parámetros del modelo no lineal con SAS para cada repetición e híbrido, los cuales fueron sometidos a un ANDEVA convencional.

El rendimiento de cada híbrido se obtuvo del asocio del peso seco de las raíces a los 139 dds por el $50 \%$ de humedad que normalmente tienen las raíces de zanahoria al momento de la cosecha y por la densidad de siembra, la cual aproximadamente fue de 900000 plantas por hectárea.

\section{RESULTADOS Y DISCUSIÓN}

\section{Fenología del cultivo de zanahoria}

La germinación, emergencia y establecimiento de las plántulas de zanahoria es un proceso lento, donde el tiempo de emergencia varía inclusive dentro de un mismo híbrido. A los 22 dds se obtuvo el $50 \%$ de la emergencia y a los 32 dds el establecimiento del cultivo y la aparición de las primeras hojas verdaderas. Lo anterior concuerda con Reid y English (2000), que informan que la germinación de la semilla y emergencia de la zanahoria puede ser extremadamente variable. Algunos factores abióticos que pueden afectar la emergencia de las plántulas 
son la preparación del terreno, la profundidad de siembra y el nivel de humedad del suelo.

La emergencia de las plántulas es clave para entender la acumulación de materia seca de las plantas de zanahoria (Benjamin 1984); una geminación diferenciada genera ventajas competitivas para las plántulas que emergen primero, ya que se afecta la densidad de siembra y la habilidad para competir por radiación, espacio, nutrimentos y agua. Lo anterior es confirmado por Benjamin (1987), que atribuye las diferencias en el tamaño de las raíces a la variación en el tiempo de emergencia, al tamaño y la madurez de la plántula.

La Figura 1 ilustra el desarrollo fenológico del cultivo de zanahoria, se utilizó el híbrido Bangor por ser el material de mayor área de siembra en el país. Según las etapas de crecimiento descritas por Bertsch (1998), en la etapa I de crecimiento, de 39 a 60 dds, se dan los primeros estados de desarrollo de la parte aérea y la raíz; en las primeras semanas la raíz crece en longitud y el desarrollo foliar es lento. En la fase II de crecimiento, de 61 a 97 dds, hay un aumento constante en el diámetro de la raíz y en el número de hojas. En la etapa III de crecimiento, de 98 a 123 dds, la tasa de crecimiento se desacelera. Finalmente, en la etapa IV, a partir de los 124 dds, el tamaño de las raíces y el follaje tiende a estabilizarse previo a la cosecha.

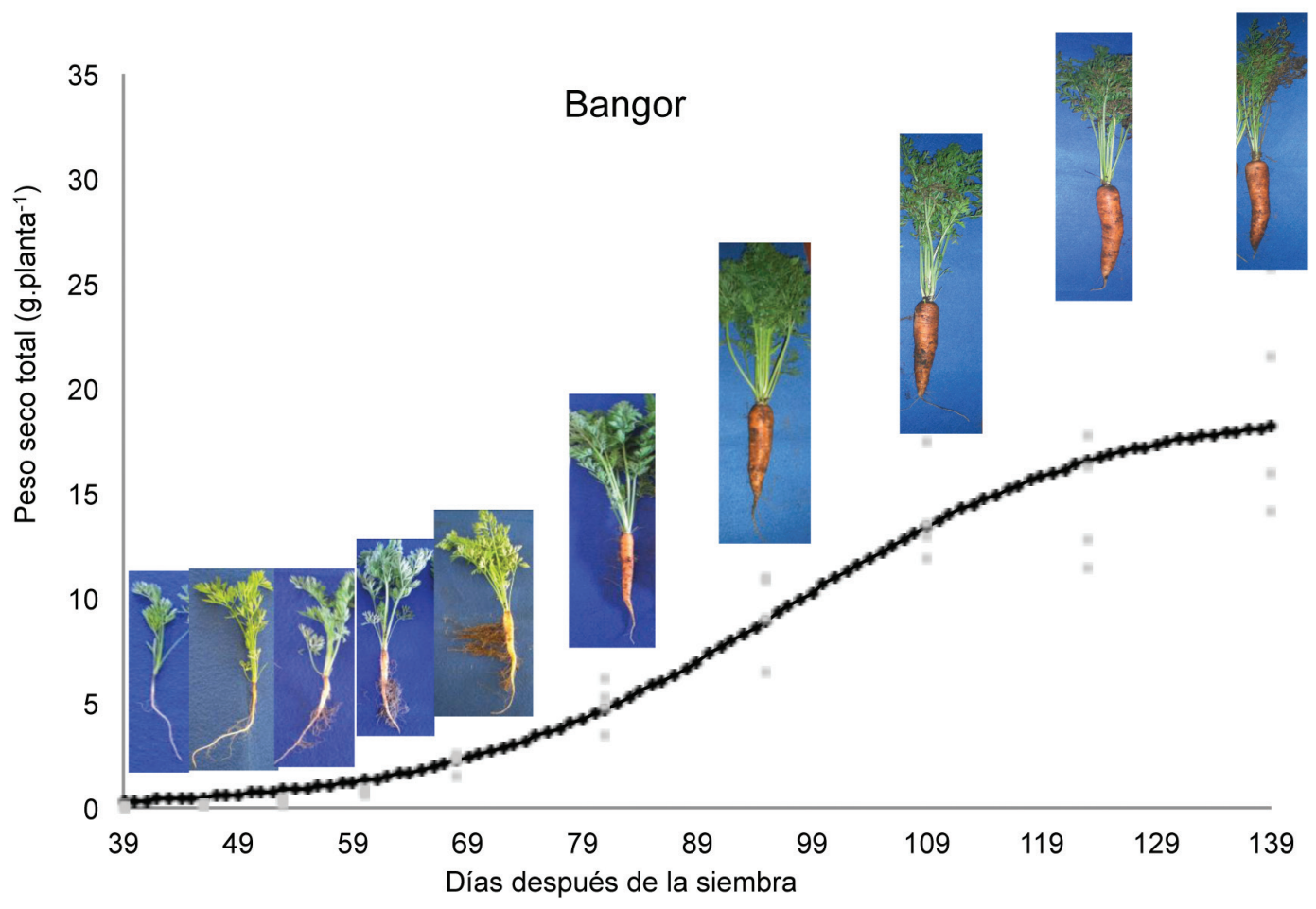

Fig. 1. Ilustración de las etapas fenológicas del cultivo de zanahoria, híbrido Bangor. Oreamuno,Cartago, 2009. 


\section{Análisis de crecimiento funcional de los híbridos de zanahoria}

La acumulación de materia seca total de los 5 híbridos en función del tiempo se muestra en la Figura 2, donde la curva de crecimiento es expresada por la línea continua que logra el mejor ajuste de los datos obtenidos en las épocas de muestreo. Para esta variable se obtuvo una función logística no-lineal asintótica.

\section{Ecuación 2}

$\mathrm{PS}=\mathrm{alfa} /(1+\exp (-$ beta $(\mathrm{x}-\mathrm{gamma})))$

Donde:

PS=Peso seco.

Alfa=el peso máximo que podría alcanzar el tejido.

Beta=la velocidad del crecimiento.

Gamma=el momento en que el crecimiento pasa de ser acelerado a desacelerado.

El crecimiento de la zanahoria es dividido en fases según la velocidad de acumulación de los fotoasimilados, y en concordancia con las etapas sugeridas por Bertsch (1998). La fase I, de 39 a $59 \mathrm{dds}$, es un periodo caracterizado por un lento aumento de materia seca y un bajo desarrollo vegetativo, lo que coincide con lo informado por Suojala (2000) y Plant Protection (2005).

La fase II, de 60 dds al valor de gamma para cada híbrido, que corresponde a los 114 dds para Suprema, 95 dds en Esperanza, 97 dds para Bangor, 82 dds en CLX-3193 y 78 dds en Sirkana. Esta fase es de crecimiento acelerado. En la fase III el crecimiento se desacelera, concluyendo esta fase a los 123 dds en Esperanza y Bangor, 109 dds en CLX-3193 y 95 dds en Sirkana.

Finalmente la fase IV de estabilidad, donde la ganancia de peso seco radical tiende a estabilizarse al aproximarse la cosecha, en concordancia con lo indicado por Plant Protection (2005). En el caso del híbrido Suprema no se logra determinar la fase IV, lo que indica que podría tratarse de un material de producción tardía. No se evalúa la etapa V sugerida por Bertsch (1998) debido a que el producto es cosechado al alcanzar el estado de desarrollo que garantiza su calidad para el mercado (madurez hortícola).

Al analizar el comportamiento de los híbridos en términos de su peso seco total es posible establecer 2 grupos, Suprema, Esperanza y Bangor por un lado, y Sirkana y CLX 3193 en otro, Figura 2. El primer grupo tuvo una mayor producción de fotoasimilados, lo cual se manifiesta con los valores superiores de alfa $(\alpha)$, Suprema 35,27 g.planta ${ }^{-1}$, Esperanza de 20,38 g.planta ${ }^{-1}$, Bangor de 19,11 g.planta ${ }^{-1}$, comparado con CLX-3193 10,65 g.planta ${ }^{-1}$ y Sirkana 6,57 g.planta ${ }^{-1}$. Igualmente los valores de gamma son mayores en el primer grupo. Las diferencias en crecimiento entre estos 2 grupos puede deberse a las características genéticas de los materiales.

La evolución del peso seco de las hojas se representa mediante una ecuación tipo "campana” y representada en la Figura 3.

\section{Ecuación 3}

$\mathrm{PS}=$ alfa*exp (-beta $\left.(\mathrm{x} \text {-gamma })^{2}\right)$

Donde:

PS=Peso seco.

Alfa=el peso máximo.

Beta=la velocidad del crecimiento.

Gamma=el tiempo en que se alcanza el peso máximo.

Del análisis de los valores de la Ecuación 3 , se observa la misma agrupación que la descrita para el peso seco total. En la variable alfa, los valores de peso máximo alcanzados son Suprema 7,51 g.planta ${ }^{-1}$, Bangor de 4,80 g.planta ${ }^{-1}$, Esperanza de 4,41 g.planta ${ }^{-1}$, y CLX-3193 de 3,92 g.planta ${ }^{-1}$ y Sirkana de 1,93 g.planta ${ }^{-1}$, en el 

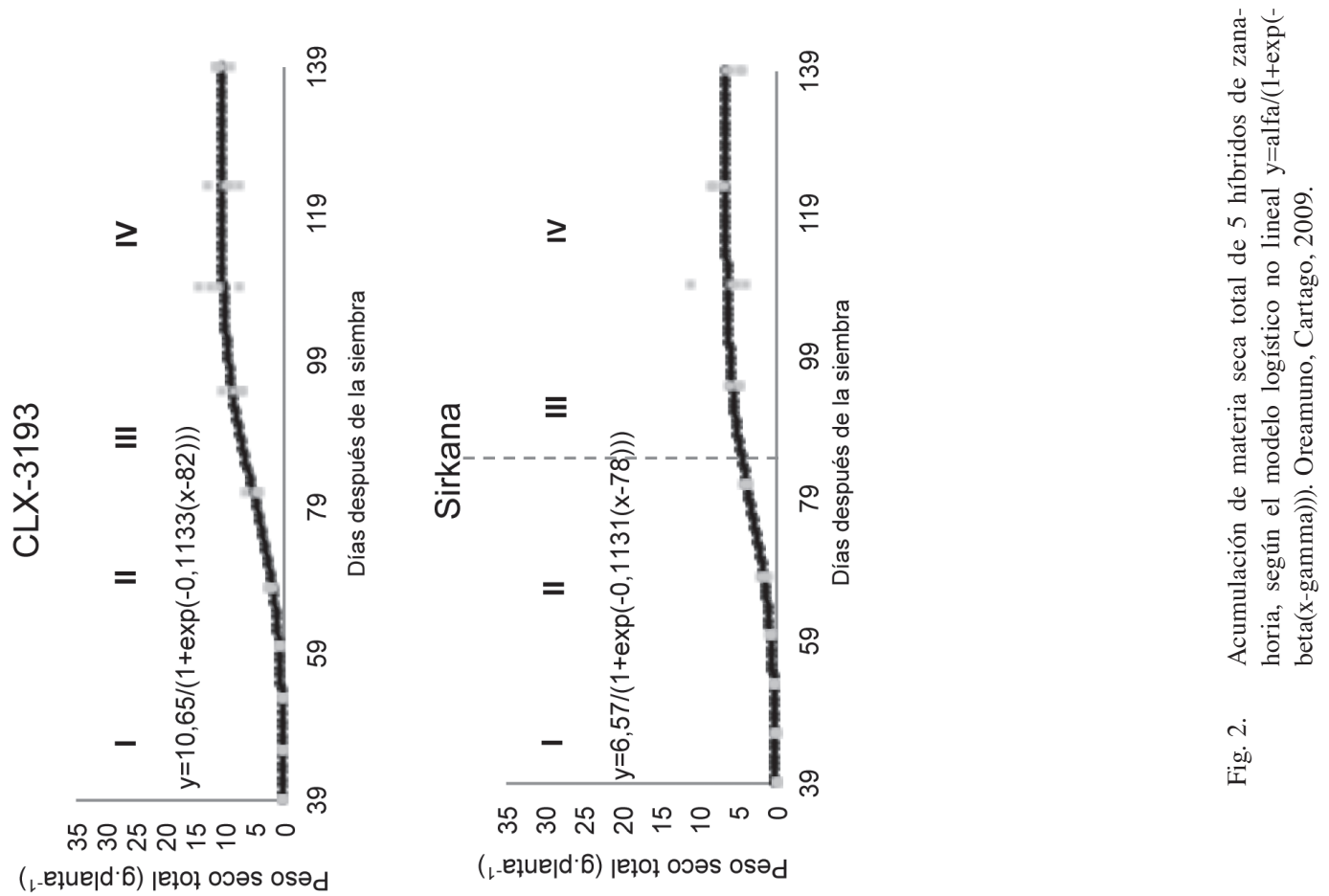

유요 유 은 눙

( (-еiuejd.6) jejol ojes osəd

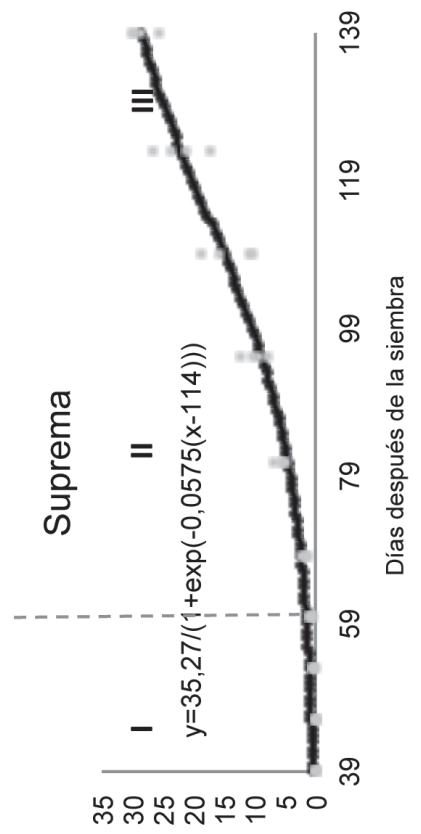

(几-ejuejd.6) jełol ojos osed
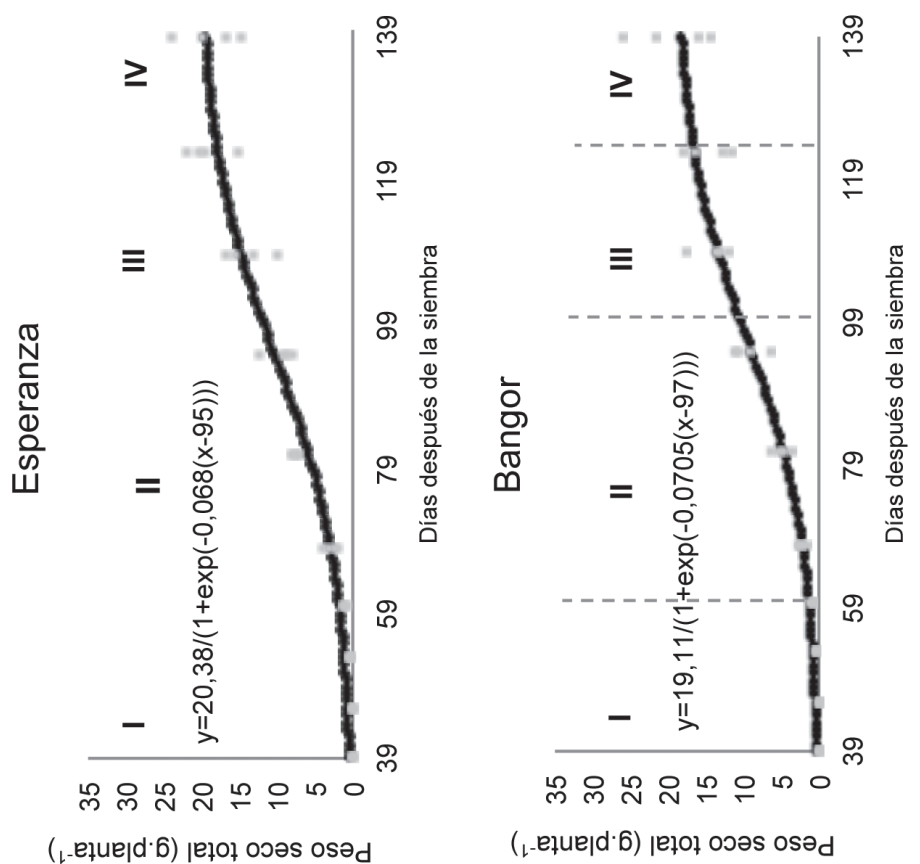


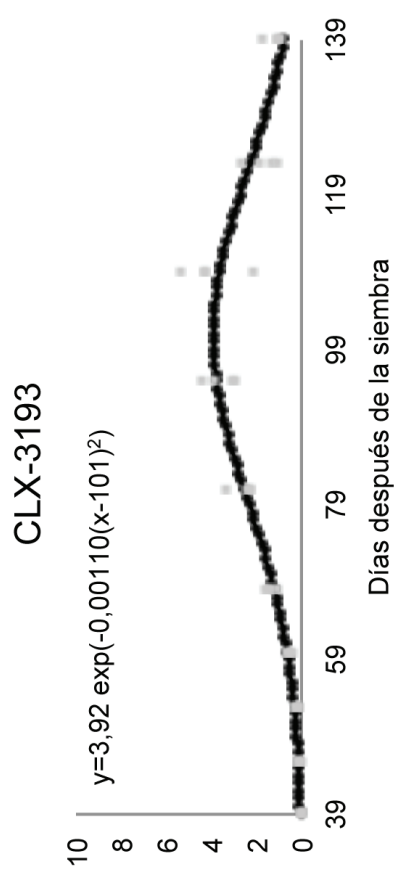

(„-ejuejd'6) seloy ojəs osəd

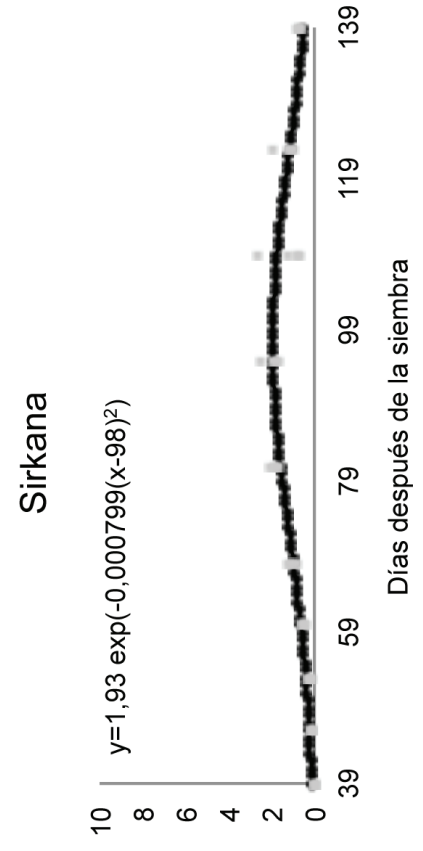

( (_-ejuejd'6) seịou ooəs osəd
总 节

킁ㅇㅇ

n :

.

'응

‡े

ช $\circ$

สู क्ष

讯

荘

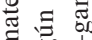

禹离

드 :

政

芯

这

$\dot{m}$

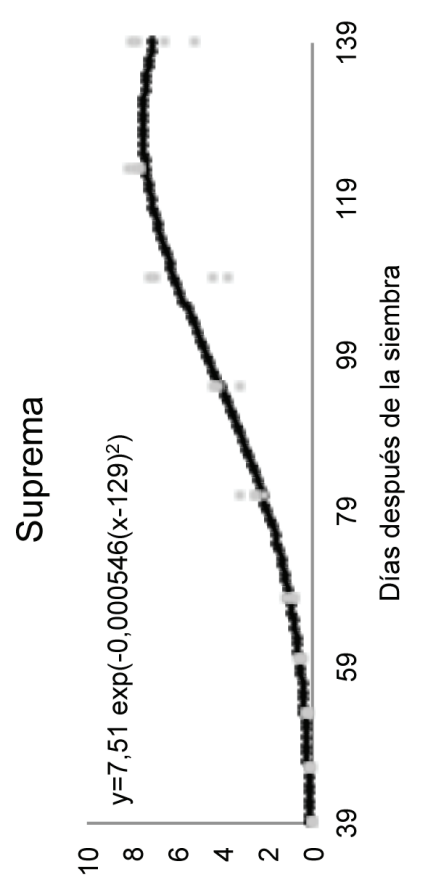

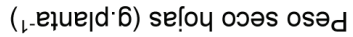

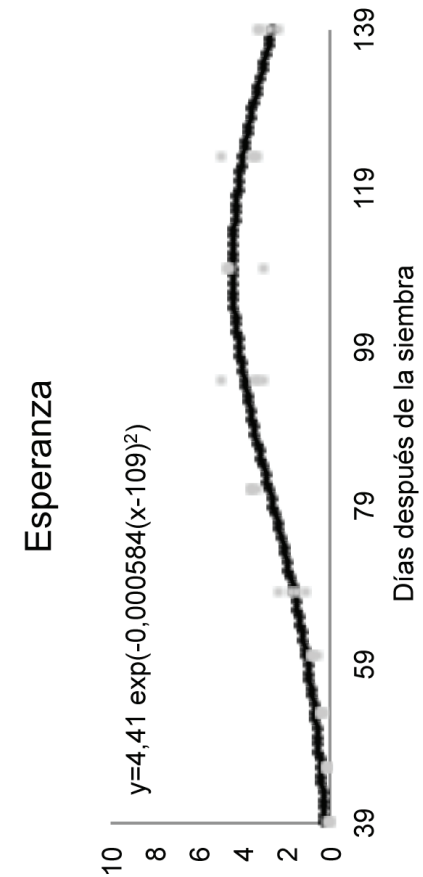

(_-eluejd.6) seloy ooəs osed

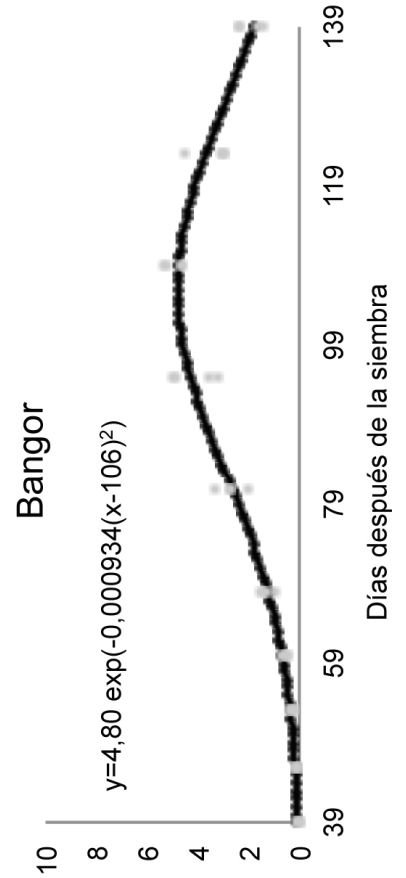

( 1 -equejd'6) se[ou osos osad 
segundo grupo. Al analizar gamma, se observa que la curva describe un crecimiento acelerado que alcanza su máximo a los 98 y 101 dds, en Sirkana y CLX-3193, respectivamente, y que es más retrasado en Bangor con 106 dds, Esperanza con 109 dds y Suprema con 129 dds. Lo anterior concuerda con Stanhill (1977) y Suojala (2000), que indican que el peso máximo del follaje se da entre los 90 y 130 dds. Para los valores de beta, velocidad de crecimiento, los híbridos Suprema, Esperanza, Bangor y CLX-3193 alcanzan su máxima acumulación en la etapa III, mientras que Sirkana lo obtiene al inicio de la fase IV.

La acumulación de materia seca de las raíces, que es también expresada por la Ecuación 2, se presenta en la Figura 4. El hecho de que las curvas de acumulación de materia seca total y de raíces tengan la misma ecuación, explica la influencia que ejerce la raíz en el desarrollo de la planta de zanahoria.

En la fase I, entre los 0 y 50 días después de emergencia (dde), la raíz de zanahoria crece rápidamente en términos de longitud, más que en términos de aumento del peso seco (Figuras $1 \mathrm{y}$ 4), lo que concuerda con Plant Protection (2005).

En la etapa II, todos los materiales tienen un crecimiento exponencial, el cual es de menor magnitud en Sirkana y en CLX-3193, comportamiento que se mantiene en las siguientes etapas, Figura 4. El modelo explica como el tejido tiene un crecimiento acelerado, fase II y III, y luego se desacelera hasta estabilizarse, lo que concuerda con lo informado por Hole et al. (1983). Así, al analizar el valor de gamma, el momento del cambio de aceleración del crecimiento, los híbridos CLX-3193 (94 dds) y Sirkana (88 dds) son los más precoces, mientras que Esperanza (104 dds), Bangor (120 dds) y Suprema (129 dds) son los más tardíos. Al examinar los valores de alfa, el peso máximo que podría alcanzar el tejido, Sirkana y CLX-3193 tienen los menores valores, 9,21 g.planta ${ }^{-1}$ y 5,6 g.planta ${ }^{-1}$, respectivamente. Mientras que fueron mayores en Suprema (33,76 g.planta $\left.{ }^{-1}\right)$, Bangor (23,02 g.planta $\left.{ }^{-1}\right)$ y Esperanza (17,94 g.planta $\left.{ }^{-1}\right)$.
El rendimiento de los híbridos en orden decreciente fue de 39 t.ha-1 para Suprema, 31 t.ha ${ }^{-1}$ para Bangor, 30 t.ha ${ }^{-1}$ para Esperanza, 16 t.ha ${ }^{-1}$ para CLX-3193 y 10 t.ha ${ }^{-1}$ para Sirkana. El efecto combinado de una baja acumulación de materia seca y una desaceleración precoz del crecimiento explican los bajos rendimientos de los híbridos CLX-3193 y Sirkana.

El desarrollo del área foliar tiene el mismo patrón y ecuación que la acumulación de materia seca de las hojas, Figura 5 y Ecuación 3. Los valores de área foliar (alfa) fueron mayores en los híbridos Suprema, Bangor y Esperanza, $7,03 \mathrm{dm}^{2} \cdot$ planta $^{-1}, 5,12 \mathrm{dm}^{2} \cdot$ planta $^{-1}$ y $4,63 \mathrm{dm}^{2}$. planta $^{-1}$, respectivamente, y bajos en CLX-3193 $\left(3,73 \mathrm{dm}^{2} \cdot\right.$ planta $\left.^{-1}\right)$ y Sirkana $\left(2,16 \mathrm{dm}^{2} \cdot\right.$ planta $\left.^{-1}\right)$. El tiempo para alcanzar máxima área foliar (gamma) también varía entre los híbridos, Sirkana a los 92 dds, CLX-3193 a los 97 dds, Bangor a los 102 dds, Esperanza a los 104 dds y Suprema a los 126 dds.

Stanhill (1977), Currah y Barnes (1979), Benjamin (1984) y Suojala (2000) informaron que el peso seco de hojas y el área foliar están correlacionados con el peso seco de las raíces, lo cual concuerda con los resultados de los híbridos Suprema y Sirkana, (Figuras 3, 4 y 5). Así, una mayor capacidad fotosintética de la planta permite una mayor acumulación de materia seca en las raíces, situación que puede atribuirse a la mayor interceptación de la radiación conforme se desarrolla el área foliar (Gardner et al. 1985). No obstante, la relación del peso entre la parte aérea y radical es afectada por factores ambientales; el crecimiento puede reducirse cuando la radiación y temperatura disminuyen (Aikman y Benjamin 1994, Hole y Sutherland 1990), o bien cuando el follaje es afectado por factores bióticos (plagas y/o enfermedades).

El híbrido Suprema alcanza los mayores valores de producción de materia seca total y de cada órgano, mientras que Sirkana obtiene los menores valores (Figuras 1, 2 y 3), la mayor producción y translocación de asimilados en Suprema puede atribuirse a un follaje de mayor magnitud y un periodo de duración más 


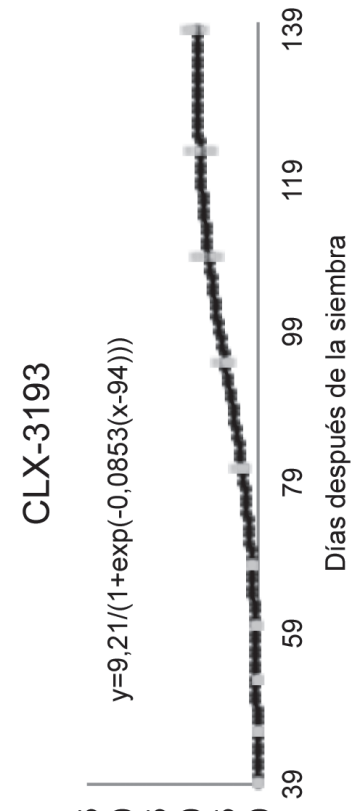

ㄴํำำㅇำ는 (几-ejuejd.6) z!ej ojəs osəd

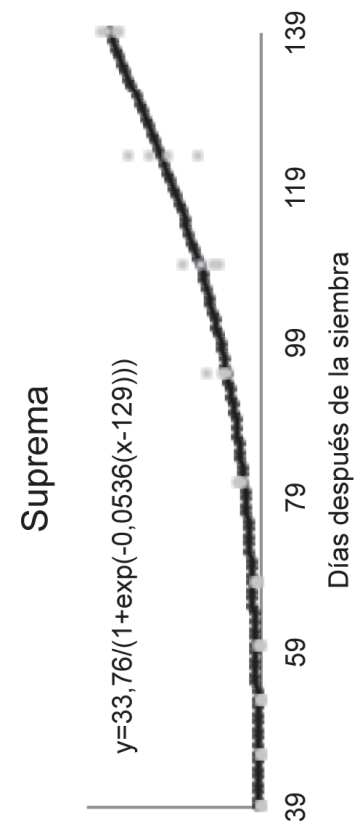

뉴윤ㅁㅇ은 ( $\_$-equejd'6) z!̣eı ooәs osəd

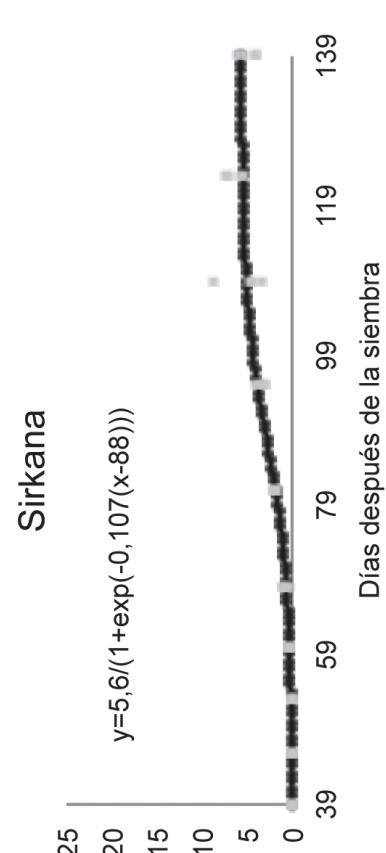

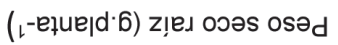

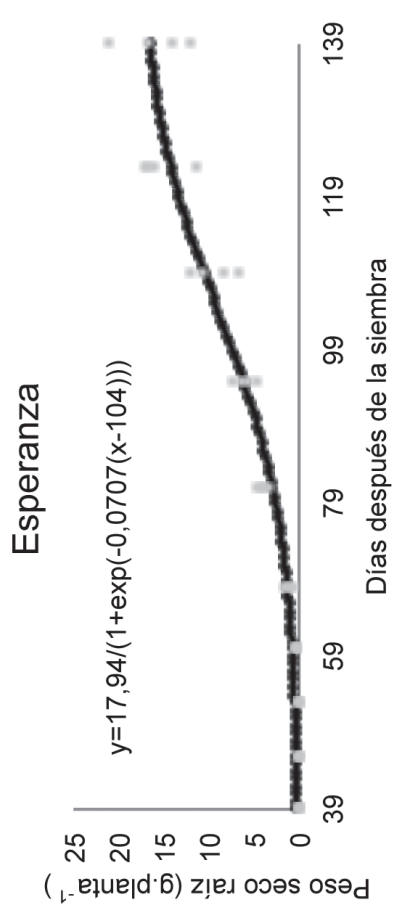

ఫิ ๘

号 III

로 ㅇํㅇ

$\div 0$ :

큰

동

$\because \stackrel{6}{0}$

芯 율

$\pi$

ฮ

$\Xi$

ช

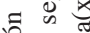

워

音

可

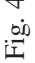




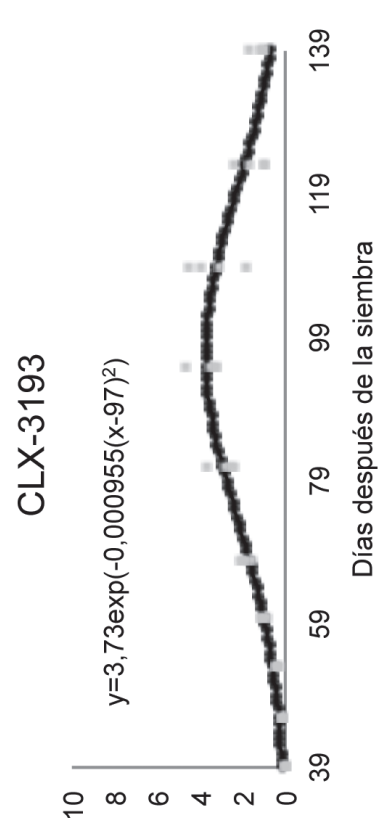

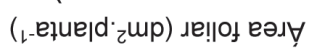

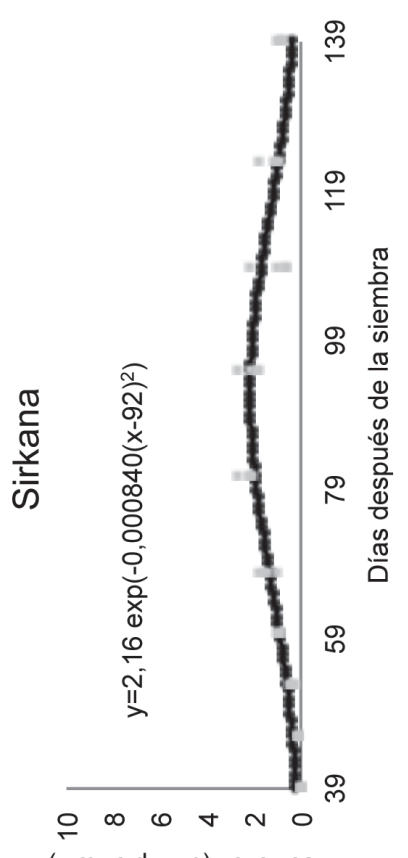

(

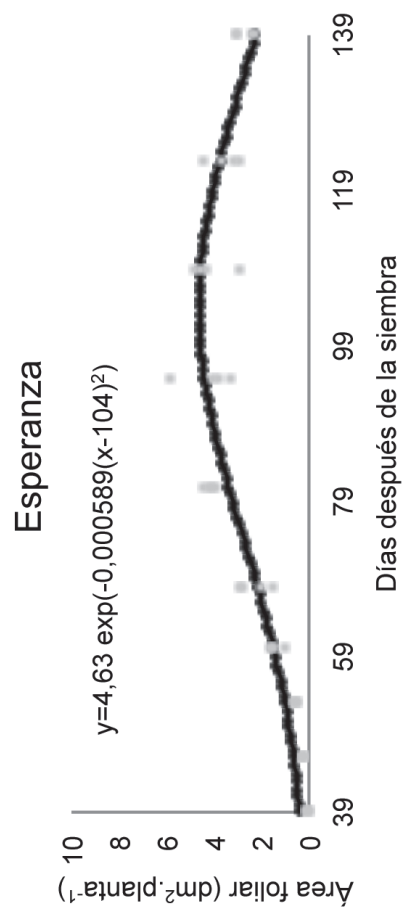

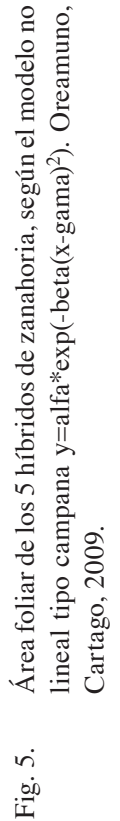

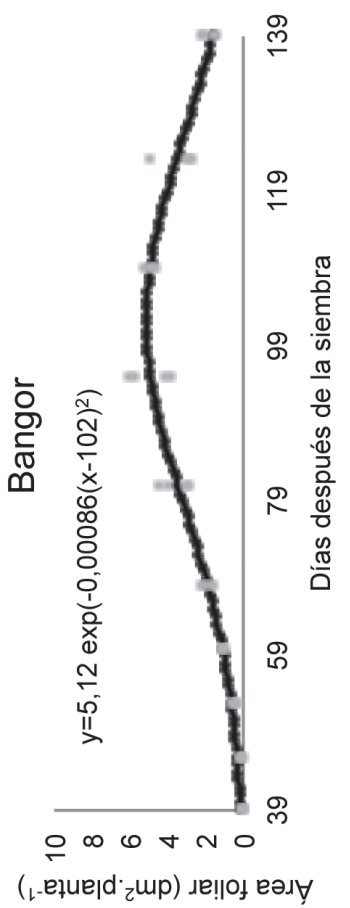


prolongado. Tirilly y Bourgeuis (2002) indican que el engrosamiento de la raíz continua mientras haya follaje activo presente.

Del análisis de la evolución del peso seco y el área foliar de los híbridos, se corrobora la separación de los híbridos en 2 grupos, Suprema, Esperanza y Bangor, y CLX-3193 y Sirkana. Suprema alcanzó los mayores valores y la desaceleración del crecimiento fue más tardía en comparación con los otros híbridos; mientras que en el segundo grupo Sirkana presentó los menores valores y alcanzó la desaceleración del crecimiento muy temprana. Si bien Suprema alcanzó valores más altos, se considera en el mismo grupo que Esperanza y Bangor ya que las raíces alcanzaron la madurez hortícola o calidad de mercado. Un mayor tiempo de acumulación de materia seca conllevaría probablemente a un mayor peso seco de raíces y total, pero no necesariamente raíces de calidad comercial.

Las diferencias de acumulación de materia seca entre los órganos de los 5 híbridos pueden atribuir a factores como el genotipo (Hole et al. 1983), las características de la semilla, factores de suelo y ambiente (Finch-Savage et al. 2001).

Los problemas bióticos también pueden ocasionar diferencias entre los híbridos, tal es el caso de la presencia de Alternaria dauci y Cercospora carotae a partir de los 99 dds. Estos patógenos mostraron mayor severidad en los híbridos Sirkana y CLX-3193, en contraste con el híbrido Suprema que presentó mayor resistencia.

La determinación de 2 modelos de crecimiento entre los híbridos es importante, ya que normalmente se asume que todos los materiales genéticos tienen un único patrón de crecimiento y que por esto debe darse el mismo manejo agronómico en términos de sistema y densidad de siembra y la nutrición, especialmente.

\section{Tasa relativa de partición de la materia seca}

La tasa relativa de partición de asimilados con respecto a la biomasa total se muestra en la Figura 6, Ecuación 1. Durante las primeras etapas de crecimiento de la zanahoria, tanto el follaje como la raíz se desarrollan simultáneamente y en sincronía. Inicialmente la parte aérea domina el crecimiento, al ser las hojas fuente y sumidero de materia seca, debido a su capacidad asimilatoria y a la inversión de energía requerida para la construcción del aparato fotosintético. Cuando el follaje alcanza un desarrollo apropiado se incrementa la movilización de materia seca hacia la raíz, lo que concuerda con Hole et al. (1983), Tirilly y Bourgeuis (2002), Krzesiński y Knaflewski (2004). Con el inicio del engrosamiento de las raíces estas se convierten en el principal sumidero de la planta y las hojas en la fuente.

En todos los híbridos de zanahoria, las raíces siempre presentan valores positivos de partición de asimilados lo que confirma su naturaleza de sumidero. Por el contrario, el follaje que inicia con valores positivos termina en negativo por su naturaleza de fuente de asimilados. No obstante, la relación del peso seco entre las partes difiere con el estado de crecimiento, diferencias que pueden ser asociadas a los cambios fisiológicos que ocurren con el inicio del proceso de engrosamiento de la raíz (Stanhill 1977, Currah y Barnes 1979).

El punto de inflexión en las curvas de la Figura 6 marca el inicio del transporte de una mayor proporción de asimilados a la raíz, momento que coincide con el crecimiento exponencial del peso total y radical, Figuras 2 y 4 , y con el engrosamiento de las raíces, Figura 1. Además, la partición foliar empieza a tener valores negativos, y sugiere que las hojas envían fotoasimilados a las raíces en lugar de acumularlos. Otra posible explicación es que las plantas estarían con pérdida de hoja por senescencia al final del ciclo.

El análisis de la partición de asimilados permite corroborar el establecimiento de los 2 grupos de híbridos, Esperanza, Suprema y Bangor, y Sirkana y CLX-3193. En el segundo grupo la duración del tiempo de partición de asimilados es menor, lo que se refleja en una caída más abrupta de la acumulación de materia seca en las hojas (Figura 6).

La distribución porcentual de materia seca entre las partes aérea y radical, durante el ciclo de cultivo, se presenta en la Figura 7. La inversión de 

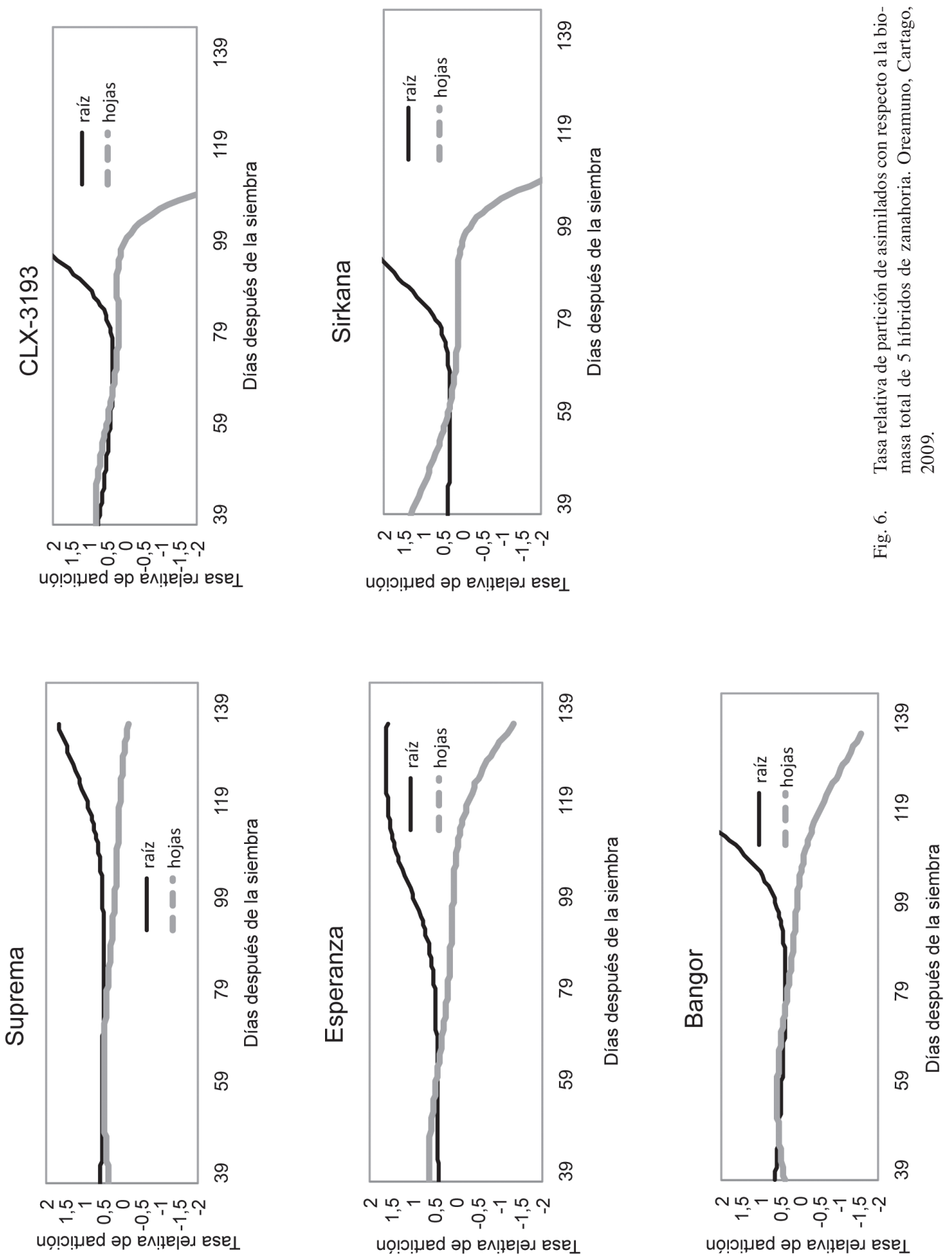

Agronomía Costarricense 36(2): 29-46. ISSN:0377-9424 / 2012 
materia seca para construir el sistema asimilatorio domina el crecimiento foliar en los primeros estados de crecimiento hasta los 59 dds, excepto en Suprema y Bangor que presentan una mayor proporción de materia seca en la raíz, sin embargo a medio ciclo se intensifica el desarrollo foliar. Sirkana manifiesta una alta inversión relativa de asimilados en el follaje en los primeros estados de desarrollo y es el único híbrido con un crecimiento porcentual sostenido de las raíces. Benjamin y Wren (1978) indican que por su condición de sumidero el sistema radical controla la producción de materia seca en la planta de zanahoria, así se observa que al final del ciclo las raíces alcanzan entre un 70 y $95 \%$ del peso seco de la biomasa total, Figura 7.

\section{Comparación entre híbridos}

El análisis de varianza para los parámetros de las funciones matemáticas de los modelos no lineales de regresión en cada variable se presentan en el Cuadro 2, los resultados permiten corroborar estadísticamente la conformación de los 2 grupos de híbridos. Suprema, Esperanza y Bangor constituyen un grupo que presenta valores estadísticamente similares en los parámetros en las variables de peso seco de raíces y área foliar, así como en beta y gamma del peso seco total.

Cuadro 2. Análisis de varianza de los parámetros que definen las funciones matemáticas de los modelos no lineales de regresión que corresponden a cada variable e híbrido de zanahoria. Oreamuno, Cartago, 2009.

\begin{tabular}{|c|c|c|c|c|c|}
\hline \multirow[b]{2}{*}{ Parámetro } & \multirow[b]{2}{*}{ Híbrido } & \multicolumn{3}{|c|}{ Peso Seco } & \multirow{2}{*}{$\begin{array}{l}\text { Área } \\
\text { Foliar }\end{array}$} \\
\hline & & Raíces & Hojas & Total & \\
\hline \multirow{5}{*}{ Alfa } & Suprema & 26,395 a & 7,6545 a & 53,86 & 7,2293 a \\
\hline & Esperanza & $17,994 \mathrm{~b}$ & $4,4341 \mathrm{~b}$ & 20,42 & $4,6616 \mathrm{bc}$ \\
\hline & Bangor & $16,952 \mathrm{~b}$ & $4,8224 \mathrm{~b}$ & 26,29 & 5,1541 b \\
\hline & CLX-3193 & $9,928 \mathrm{c}$ & $4,0287 \mathrm{~b}$ & 10,76 & $3,9107 \mathrm{c}$ \\
\hline & Sirkana & $5,614 \mathrm{c}$ & $2,0098 \mathrm{c}$ & 6,54 & $2,2541 \mathrm{~d}$ \\
\hline \multicolumn{2}{|c|}{$\% \mathrm{CV}$} & 15 & 12 & 92 & 12 \\
\hline \multirow{5}{*}{ Beta } & Suprema & $0,0659 \mathrm{~b}$ & $0,0005560 \mathrm{~b}$ & $0,05980 \mathrm{~b}$ & $0,0004818 \mathrm{~b}$ \\
\hline & Esperanza & $0,0711 \mathrm{~b}$ & $0,0005913 \mathrm{~b}$ & $0,07013 \mathrm{~b}$ & $0,0005973 \mathrm{ab}$ \\
\hline & Bangor & $0,0695 \mathrm{~b}$ & $0,0009510 \mathrm{ab}$ & $0,07210 \mathrm{~b}$ & $0,0008793 \mathrm{ab}$ \\
\hline & CLX-3193 & $0,0884 \mathrm{ab}$ & 0,0011843 a & 0,11790 a & 0,0010143 a \\
\hline & Sirkana & 0,1077 a & $0,0009120 \mathrm{ab}$ & 0,12300 a & 0,0010010 a \\
\hline \multicolumn{2}{|c|}{$\% \mathrm{CV}$} & 14 & 23 & 22 & 28 \\
\hline \multirow{5}{*}{ Gamma } & Suprema & $187,7 \mathrm{c}$ & 199,950 a & 193,48 a & $197,600 \mathrm{a}$ \\
\hline & Esperanza & $174,7 \quad b$ & $180,225 \mathrm{~b}$ & $164,83 \mathrm{ab}$ & $174,825 \mathrm{~b}$ \\
\hline & Bangor & $179,8 \mathrm{bc}$ & 177,075 bc & $176,88 \mathrm{ab}$ & $173,275 \mathrm{~b}$ \\
\hline & CLX-3193 & $166,2 \mathrm{a}$ & $171,800 \mathrm{bc}$ & $152,78 \mathrm{ab}$ & $167,875 \mathrm{bc}$ \\
\hline & Sirkana & 158,8 a & $167,875 \mathrm{c}$ & $148,33 \mathrm{~b}$ & $162,275 \mathrm{c}$ \\
\hline \multicolumn{2}{|c|}{$\% \mathrm{CV}$} & 2 & 2 & 11 & 3 \\
\hline
\end{tabular}




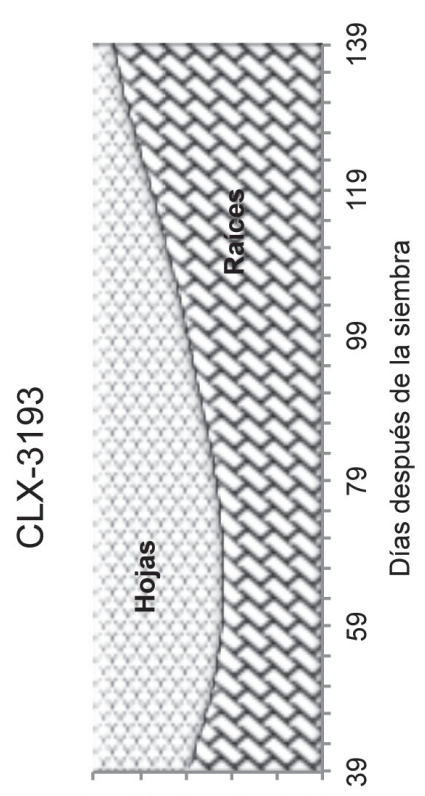

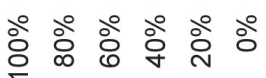
ojes osəd

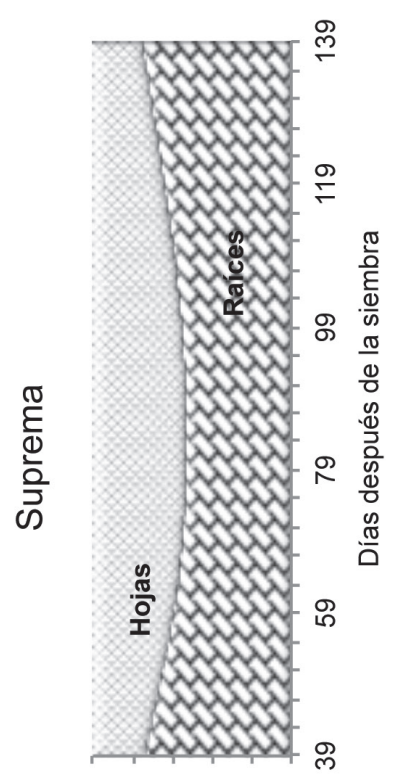

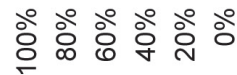
oวəs osəd

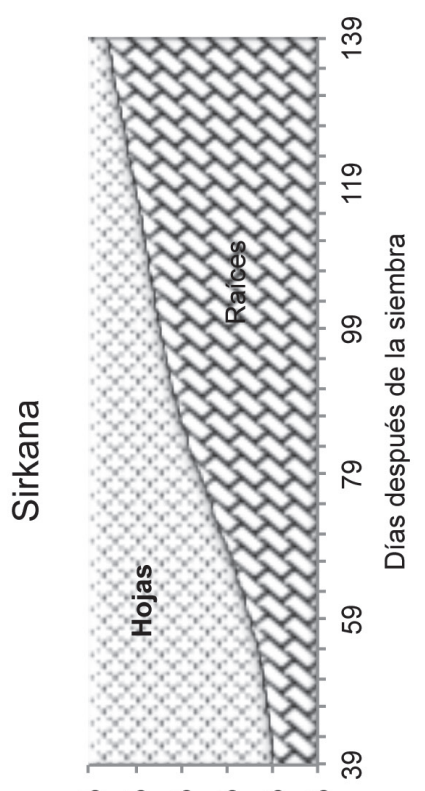

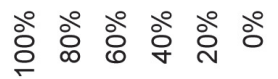
oग्S osəd

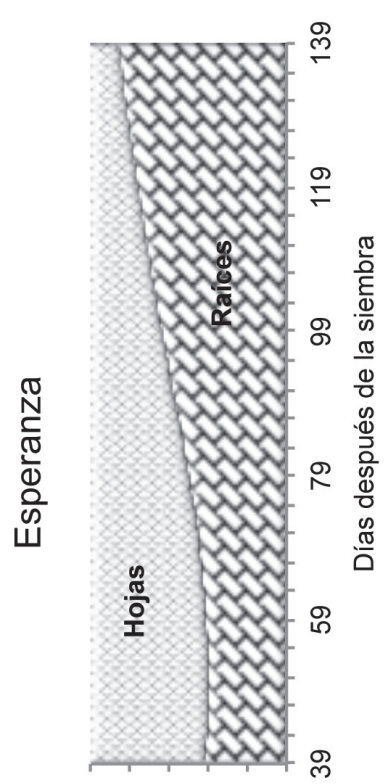

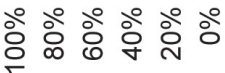
oग्र osəd

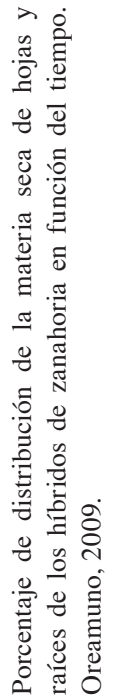

$\therefore$

in

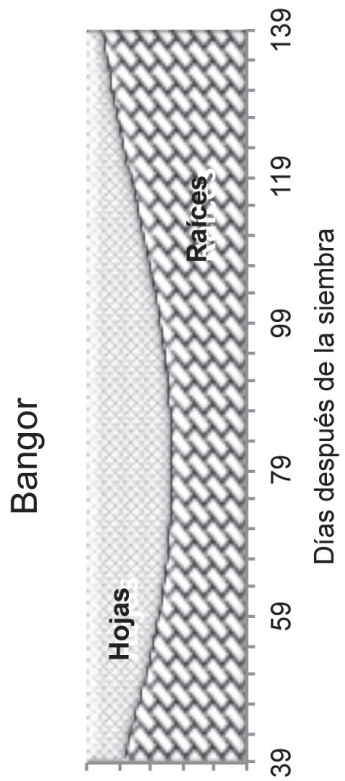

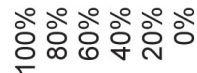
ojes osəd 


\section{CONCLUSIONES}

Los 5 híbridos presentaron una curva de acumulación de materia seca de tipo sigmoidal, con 4 fases bien definidas: una primera etapa de crecimiento lento, una fase de crecimiento exponencial, seguido por una fase de desaceleración del crecimiento y por último un periodo de estabilidad, dichas etapas fisiológicas concuerdan con la fenología del cultivo.

La raíz de la zanahoria comienza a engrosar según el híbrido durante la etapa II de crecimiento, el inicio del engrosamiento determina una mayor partición de asimilados a la raíz, lo que conduce al aumento en su diámetro. Previamente, la planta se dedica a desarrollar su sistema fotosintético en comparación con el desarrollo radical, durante esta etapa la raíz crece aceleradamente en términos de longitud.

Se puede clasificar los híbridos en 2 grupos basados en el comportamiento de las variables de peso seco, área foliar y partición de asimilados: Grupo 1. Suprema, Esperanza y Bangor, y Grupo 2. CLX-3193 y Sirkana.

Las diferencias en las variables de crecimiento que se presentaron entre los 5 híbridos pueden deberse a la interacción de factores como el genotipo, el ambiente, las características de la semilla, el tiempo de emergencia, el manejo de la plantación, la densidad de siembra y la diferencia en la susceptibilidad a enfermedades como Alternaria dauci y Cercospora carotae.

\section{LITERATURA CITADA}

AIKMAN D.P., BENJAMIN L.R. 1994. A model for plant and crop growth, allowing for competition for light by the use of potential and restricted projected crown zone areas. Annals of Botany 73:185-194.

BENJAMIN L.R. 1984. Role of foliage habit in the competition between differently sized plants in carrots crops. Annals of Botany 53:549-557.

BENJAMIN L.R. 1987. Variation in plant size and the timing of carrot production. Acta Horticulturae. 198:297-304.

BENJAMIN L.R., McGARRY A., GRAY D. 1997. The root vegetables: beet, carrot, parsnip and turnip, pp. 553-580. In: H.C. Wien (ed.). The physiology of vegetable crops. CAB Internacional.
BENJAMIN L.R., WREN M.J. 1978. Root development and source-sink relations in carrot, Daucus carota. Journal of Experimental Botany 29:425-433.

BERTSCH F. 1998. La fertilidad de los suelos y su manejo. San José, Costa Rica, Asociación Costarricense de las Ciencias del Suelo. $157 \mathrm{p}$.

CURRAH I.E., BARNES A. 1979. Vegetable plant part relationships. I. Effects of time and population density on the shoot and storage root weights of carrot (Daucus carota L.). Annals of Botany 43:475-486.

FINCH-SAVAGE W.E., PHELPS K., STECKEL J.R.A., WHALLEY W.R., ROWSE H.R. 2001. Seed reserve-dependent growth responses to temperature and water potential in carrot (Daucus carota L.). Journal of Experimental Botany 52(364):2187-2197.

GARDNER F.P., PEARCE R.B., MITCHELL R.L. 1985. Carbon fixation by crop canopies, pp. 31-57. In: Physiology of Crop Plants. Ames, Iowa State University Press.

HOLE C.C., BARNES A., THOMAS T.H., SCOTT P.A., RANKIN W.E.F. 1983. Dry matter distribution between the shoot and the storage root of carrot (Daucus carota L.). I. Comparison of varieties. Annals of Botany 51:175-187.

HOLE C.C., DEARMAN J. 1991. Carbon economy of carrots during initiation of storage root in cultivars contrasting in shoot: root ratio at maturity. Annals of Botany 68:427-434.

HOLE C.C., SUTHERLAND R.A. 1990. The effect of photon flux density and the duration of the photosynthetic period on growth and dry matter distribution in carrot. Annals of Botany 65:63-69.

HUNT R. 2003. Growth analysis, individual plants, pp. 588596. In: B. Thomas, D.J. Murphy and D. Murray (eds.). Encyclopedia of applied plant sciences. Academic Press, London.

KRZESIŃSKI W., KNAFLEWSKI M. 2004. Preliminary Model of Carrot Growth. Acta Horticulturae 654:235-242.

PLANT PROTECTION. 2005. Zanahoria. Programa Leonardo da Vinci de la Comisión Europea. Consultado 14 setiembre 2007. Formato HTML. Disponible en http://www.plantprotection.hu/ modulok/spanyol/root_veg/growth_root.htm

REID J.B., ENGLISH J.M. 2000. Potential yield in carrots (Daucus carota L.): theory, test and an application. Annals of Botany 85:593-605.

RICHMOND F.J. 2009. Evaluación agronómica de 12 cultivares comerciales de zanahoria (Daucus carota L.) en Cot, Cartago. Tesis de licenciatura en Ingeniería Agronómica. Universidad de Costa Rica. San José, Costa Rica. 91 p.

RODRÍGUEZ W., LEIHNER D. 2006. Análisis del crecimiento vegetal. Volumen 7 de la Serie: Fisiología de la producción de los cultivos tropicales. 
Primera Edición. Editorial Universidad de Costa Rica. San José, Costa Rica. 37 p.

ROJAS M., ROVALO M. 1985. Fisiología vegetal aplicada. Tercera Edición. McGraw-Hill. Mexico. 302 p.

STANHILL G. 1977. Allometric growth studies of the carrot crop. I. Effects of plant development and the cultivar. Annals of Botany 41:533-540.

SUOJALA T. 2000. Pre and postharvest development of carrot yield and quality. University of Helsinki. Department of Plant Production. Section of Horticulture. Publication No. 37. Helsinki. 43 p.

TIRILLY Y., BOURGEUIS C.M. 2002. Tecnología de las hortalizas. Editorial Arcribia, S.A. Zaragoza, España. 591 p.
VALADEZ A. 1998. Producción de Hortalizas. Editorial Limusa, S.A. México. 109-115 pp.

VENUS J.C., CAUSTON D.R. 1979. Plant growth analysis: a re-examination of the methods of calculation of relative growth and net assimilation rates without using fitted functions. Annals of Botany 43:633-638.

WESTERVELD S.M., McKEOWN A.W., McDONALD M.R. 2006. Seasonal nitrogen partitioning and nitrogen uptake of carrots as affected by nitrogen application in a mineral and an organic soil. HortScience 41(5):1332-1338. 